\title{
Relação entre Inteligência, Criatividade, Personalidade e Superdotação no Contexto Escolar
}

\author{
Marina Nogueira ${ }^{1}$ \\ ${ }^{1}$ Universidade de Brasília, Brasília, DF, Brasil.
}

\author{
Denise de Souza Fleith ${ }^{1}$ \\ ${ }^{1}$ Universidade de Brasília, Brasília, DF, Brasil.
}

\begin{abstract}
Resumo: As diferenças entre indivíduos superdotados e não superdotados vêm sendo alvo de muitos estudos e controvérsias. Este estudo teve como objetivo comparar alunos que ingressaram precocementenauniversidade (grupo I), alunos que ingressaram na universidade no tempo regular (grupo II) e alunos superdotados do ensino médio (grupo III) a respeito da inteligência, criatividade e personalidade, bem como investigar a relação entre essas variáveis. Participaram do estudo 30 alunos (15 de cada sexo), com média de idade de 17,3 anos. Foram utilizados instrumentos de inteligência (Matrizes Progressivas de Raven), criatividade (Teste Torrance de Pensamento Criativo) e personalidade (Inventário Fatorial de Personalidade e Rorschach), e os dados foram tratados com análises não paramétricas de Kruskal-Wallis, Mann-Whitney e correlação de Spearman. Os resultados apontaram diferenças significativas em relação à inteligência $\left(X^{2}[2, \mathrm{~N}=30]=5,975\right.$; $p=0,05)$ e aos fatores de criatividade: fluência $\left(X^{2}[2, \mathrm{~N}=30]=6,302 ; p=0,043\right)$ e flexibilidade $\left(X^{2}[2, \mathrm{~N}=30]=6,415 ; p=0,040\right)$. Observou-se correlação positiva entre inteligência e o fator analogia $\left(r_{s}=0,426 ; p=0,019\right)$. Os alunos dos grupos I e III demonstraram vantagens nos fatores de inteligência e criatividade em relação aos do grupo II, e os do grupo I demonstraram ainda maior abertura a novas experiências e mudanças do que os alunos dos outros dois grupos. Os resultados apontam que inteligência e criatividade são construtos diferentes, mas relacionados, sugerindo que inteligência seria um subconjunto de criatividade.
\end{abstract}

Palavras-chave: Inteligência; Personalidade; Criatividade; Superdotação; Aceleração escolar.

\section{Association of Intelligence, Creativity, Personality, and Giftedness in the School Context}

\begin{abstract}
Several studies have addressed the differences between gifted and non-gifted individuals, generating much controversy. This study aimed to investigate the association between intelligence, creativity, and personality, and compare these variables in three groups of students: group I, including early entrants to university; group II, including regular entrants; ang group III, high school students enrolled in a gifted program (group III). Thirty students (15 males and 15 females) with mean age of 17.3 years participated in the study. Intelligence was measured using the Raven's Progressive Matrices, creativity with the Torrance Test of Creative Thinking, and personality with the Factorial Personality Inventory and Rorschach. Data were analyzed using non-parametric Kruskal-Wallis, Mann-Whitney, and Spearman's correlations tests. The results showed significant differences regarding intelligence $\left(X^{2}[2, \mathrm{~N}=30]=5,975 ; p=0,05\right)$ and the creative factors fluency $\left(X^{2}[2, \mathrm{~N}=30]=6,302 ; p=0,043\right)$ and flexibility $\left(X^{2}[2, \mathrm{~N}=30]=6,415 ; p=0,040\right)$, besides indicating a positive correlation between intelligence and analogy $\left(r_{s}=0,426 ; p=0,019\right)$. When compared to group II students, those of groups I and III showed advantages in intelligence and creativity. Moreover, group I students also showed a greater openness to new experiences and changes when compared to those of the two other groups. The results indicate that intelligence and creativity are different but related constructs, suggesting that intelligence would be a subset of creativity.
\end{abstract}

Keywords: Intelligence; Personality; Creativity; Giftedness; School acceleration. 


\title{
Relación entre Inteligencia, Creatividad, Personalidad y Superdotación en el Contexto Escolar
}

\begin{abstract}
Resumen: Las diferencias entre individuos superdotados y no superdotados son objeto de diversos estudios y controversias. Este estudio tuvo como objetivo comparar alumnos que ingresaron precozmente a la universidad (grupo I), alumnos que ingresaron a la universidad en el tiempo regular (grupo II), y alumnos superdotados de la secundaria (grupo III) en relación a inteligencia, creatividad y personalidad, así como investigar la relación entre esas variables. Participaron en el estudio 30 alumnos (15 de cada sexo) con edad media de 17,3 años. Los instrumentos utilizados fueron los de inteligencia (Matrices Progresivas de Raven), de creatividad (Test de Pensamiento Creativo de Torrance) y de personalidad (Cuestionario Factorial de Personalidad y Rorschach); para procesar los datos se utilizó pruebas no paramétricas de Kruskal-Wallis, de Mann-Whitney y la correlación de Spearman. Los resultados mostraron diferencias significativas en relación a inteligencia $\left(X^{2}[2, \mathrm{~N}=30]=5,975 ; p=0,05\right)$ y en los factores de creatividad: fluencia $\left(X^{2}[2, \mathrm{~N}=30]=6,302 ; p=0,043\right)$ y flexibilidad $\left(X^{2}[2, \mathrm{~N}=30]=6,415 ; p=0,040\right)$. Hubo una correlación positiva entre inteligencia y el factor analogía $\left(r_{s}=0,426 ; p=0,019\right)$. Los estudiantes de los grupos I y III demostraron ventajas en los factores de inteligencia y creatividad en relación al grupo II, y los del grupo I mostraron mayor apertura a nuevas experiencias y cambios en comparación a los otros dos grupos. Los resultados indican que la inteligencia y la creatividad son constructos distintos pero relacionados, sugiriendo que la inteligencia sería un subconjunto de la creatividad.
\end{abstract}

Palabras clave: Inteligencia; Personalidad; Creatividad; Superdotación; Aceleración escolar.

\section{Introdução}

É recorrente, na história do mundo, a existência de pessoas que se destacam como muito talentosas em alguma área, gerando produtos e ideias que romperam paradigmas de sua época. Em menor escala, também é muito comum encontrar, no dia a dia, pessoas que se sobressaem no ambiente escolar ou de trabalho por sua inteligência, criatividade ou personalidade. Esses indivíduos passaram a ser estudados não apenas com o objetivo de compreender o que os diferenciava dos demais, mas também de identificar caminhos para o desenvolvimento de suas potencialidades (Nogueira \& Fleith, 2019; Renzulli, 2016a).

Essas pessoas são denominadas indivíduos com altas habilidades/superdotação (AH/SD) e, segundo o Modelo dos Três Anéis de Renzulli (1978, 2002, 2005, 2016b), o fenômeno pode ser entendido a partir da interação de três variáveis: habilidade acima da média, criatividade e envolvimento com a tarefa, compreendidas dentro de um determinado contexto social.

Habilidade acima da média envolve habilidades gerais e específicas. As primeiras se referem à capaci- dade de processar informações, integrar experiências que resultam em respostas adaptativas e apropriadas a novas situações e na capacidade de se engajar em pensamento abstrato. Geralmente são medidas por testes de aptidão e de inteligência. As habilidades específicas tratam da capacidade de adquirir conhecimento e atuar em uma ou mais atividades de uma área especializada. Essas habilidades podem ser identificadas na forma como o indivíduo se expressa na vida real, e não em situação de avaliação ou testagem.

Criatividade envolve curiosidade, sensibilidade, pensamento independente e divergente, e envolvimento com a tarefa diz respeito à motivação envolvida na execução da atividade ou resolução de um problema. Além disso, alguns estudos (Ismail, Anuar, \&Yusuf, 2020; Ourofino \& Guimarães, 2007; Renzulli, 1978, 2016b) descrevem que indivíduos com altas habilidades teriam personalidade mais extrovertida, aberta a novas experiências, imaginativa e independente, além de serem perfeccionistas, sensíveis, perseverantes e autoconscientes.

Gonçalves e Fleith (2011) realizaram um estudo para examinar se havia diferenças entre grupos de 
alunos superdotados e não superdotados em relação à inteligência e criatividade, mensuradas respectivamente pelas Matrizes Progressivas de Raven e Teste Torrance de Pensamento Criativo, e, ainda, se esses dois construtos apresentavam alguma correlação. Não foram encontradas correlações significativas entre criatividade e inteligência em nenhum dos grupos e tampouco diferenças entre eles com relação à inteligência. Já no tangente à criatividade, percebeu-se diferença significativa entre os dois grupos no fator originalidade, sendo que os alunos superdotados apresentaram maiores médias do que os alunos não superdotados. As autoras destacaram que o teste de inteligência utilizado avalia apenas o aspecto do raciocínio analógico da inteligência, isso é, a capacidade de observação e clareza de pensamento, sugerindo que os superdotados apresentariam diferenças em relação a seus pares não superdotados em outras facetas desse construto.

Por outro lado, ao aplicarem a Escala Wechsler de Inteligência IV (WISC-IV), que mensura velocidade de processamento, memória de curto prazo, inteligência cristalizada, conhecimento quantitativo e processamento visual da inteligência, Macedo, Mota e Mettrau (2017) encontraram diferenças significativas entre os quocientes intelectuais (QI) dos grupos de superdotados e não superdotados, tendo o primeiro obtido maior média. Em um estudo comparando os níveis de habilidade intelectual e potencial criativo em grupos de superdotados e não superdotados, Guignard, Kermarrec e Tordjman (2016) encontraram correlações fracas ou nulas entre inteligência, avaliada pelo WISC-IV, e criatividade, medida pelo instrumento Evaluation of Potential Creativity (EPoC). Os achados revelaram, ainda, que os dois grupos não diferiram em relação à criatividade, sugerindo que alta inteligência não contribui, necessariamente, para o processo criativo. Conclui-se, a partir dos estudos mencionados, que os resultados acerca da relação criatividade e inteligência não são conclusivos. Vale lembrar que autores como Sternberg $(1999,2005) \mathrm{e}$ Gardner (1983) ampliaram a concepção de inteligência, propondo uma visão multidimensional do fenômeno, na qual os indivíduos podem se desenvolver em algumas dimensões, mas não necessariamente em outras.

Zeidner e Shani-Zinovich (2011) conduziram um estudo para verificar se havia diferenças entre um grupo de superdotados e um de não superdotados em relação aos fatores de personalidade descritos pela Teoria dos Cinco Grandes Fatores. Eles concluíram que o grupo de alunos superdotados apresentou escores mais elevados no fator abertura a experiências, mas escores inferiores nos fatores neuroticismo e socialização. Nos demais fatores - extroversão e realização -, não foram encontradas diferenças significativas entre os grupos. Os autores investigaram, ainda, as diferenças entre os dois grupos em relação à ansiedade $\mathrm{e}$ saúde mental, em termos de estresse e bem-estar na vida cotidiana. Os resultados apontaram que, apesar de evidências de que os superdotados apresentam menores índices de ansiedade, não houve diferença entre os grupos nos fatores de estresse e bem-estar

Devido às diferenças e peculiaridades dos alunos superdotados, um dos aspectos que tem sido muito destacado por autores da área (e. g. Azevedo \& Mettrau, 2010; Renzulli, 2016a; Sabatella \& Cupertino, 2007) é a necessidade de um atendimento correto aos alunos superdotados, que considere suas particularidades acadêmicas, sociais e emocionais. Uma das formas de se atender às necessidades educacionais desses alunos é a aceleração de estudos, que consiste em cumprir o programa escolar em menos tempo, provendo ao aluno oportunidades de se envolver com atividades desafiadoras, de modo que a escola não se torne um lugar enfadonho. Contudo, uma série de mitos acerca do desenvolvimento cognitivo, emocional e social permeiam essa prática. Um dos mais difundidos é a crença de que a pessoa que foi acelerada não irá acompanhar social e emocionalmente seus colegas mais velhos, podendo enfrentar dificuldades de ajustamento social e emocional.

No entanto, como alguns autores apontaram (Alencar, 2007; Brasil, 2006), os alunos superdotados costumam ter preferência por conversar com pessoas mais velhas ou com idade mental semelhante a sua, já que as pessoas de sua idade costumam não compartilhar dos mesmos interesses que eles. Além disso, pesquisas têm apontado que o ajustamento social de pessoas superdotadas muitas vezes é melhor do que o de seus pares não superdotados (Alencar, 2007). Segundo Brody, Muratori e Stanley (2004), a maioria dos estudos com participantes que tiveram ingresso precoce na universidade revela que eles não apenas obtiveram bons resultados acadêmicos, como não demonstraram dificuldades de ajustamento social ou emocional. Alencar (2007) destaca uma pesquisa realizada por Gross cujos resultados indicaram que os alunos com habilidades acadêmicas superiores que não foram acelerados tiveram mais dificuldades de se ajustar socialmente e índices mais baixos de 
motivação e autoestima. Conclui-se que a aceleração pode instigar no aluno a curiosidade, ampliação e aprofundamento do conhecimento, impedindo que crie hábitos inadequados de estudo ou, ainda, desinteresse em aprender.

Acredita-se ainda que, se a pessoa avança uma série, por exemplo, ficará em desvantagem em relação aos demais alunos, pois não terá todo o conteúdo que outras crianças tiveram. No entanto, a criança que avança de série ou cujo currículo é compactado, isso é, cujo currículo regular é adaptado (substituindo-se os conteúdos que o aluno já domina por outros mais desafiadores), já tem domínio dos conteúdos do currículo regular que serão passados ao longo daquele ano. Se a escola for um ambiente pouco desafiador para a criança, pode gerar sub-rendimento e evasão escolar, culminando em frustrações para ela e para a família e cobranças por parte da escola (Alencar, 2007).

Os resultados do estudo de Reis, Westberg, Kulikowich e Purcell (2016) corroboraram a literatura da área, demonstrando que, muitas vezes, os alunos que passaram pela compactação curricular apresentam melhores resultados com relação ao rendimento acadêmico, enfatizando a importância de um currículo adequado ao ritmo de aprendizagem do aluno. Brody, Assouline e Stanley (1990) compararam o rendimento acadêmico de 65 alunos que ingressaram precocemente na universidade com o de seus pares que ingressaram no tempo regular, e perceberam que não só os alunos acelerados se graduavam com menor tempo, como também recebiam mais prêmios durante a graduação.

De acordo com o Modelo dos Três Anéis (Renzulli, 2016b), um dos tipos de superdotação é a acadêmica, muito valorizada no contexto tradicional de aprendizagem. Os alunos que apresentam esse comportamento aprendem rapidamente, têm um excelente rendimento acadêmico, tendem a ser facilmente identificados em testes padronizados de inteligência ou aptidão e se beneficiam de adaptações curriculares, como a aceleração.

Não se pode afirmar, contudo, que a aceleração seria benéfica em todos os casos. Cada um deve ser analisado cuidadosamente, levando em conta as características pessoais da criança, a possibilidade da família de dar suporte às necessidades dela e a maneira como isso seria amparado prática e teoricamente pela escola e pelos profissionais que a receberiam. Evidencia-se que, para lidar com a educação da criança superdotada, não basta levar em conta ape- nas suas características cognitivas. As características sociais e emocionais são igualmente relevantes, pois se a criança estiver sendo muito pressionada ou sentindo que não é capaz de alcançar a expectativa dos pais e da escola, poderá perder o interesse em investir no próprio potencial.

Muitas são as características socioemocionais das crianças superdotadas, que tanto podem ser um estímulo ao seu potencial quanto podem configurar fonte de ansiedade. Cross e Andersen (2016), Cross e Cross (2015) e Pfeiffer (2013) elencam algumas dessas características: a) idealismo e senso de justiça aguçados; b) perfeccionismo; c) alto nível de energia envolvido na realização da atividade; d) inconformismo; e) sensibilidade às expectativas dos outros; $\mathrm{f}$ ) intensidade e sensibilidade emocional; g) consciência aguçada de si mesmo; h) altas expectativas em relação a si mesmo e aos outros. Considerar esses aspectos emocionais e sociais, para além dos cognitivos, é importante no processo de identificação, no planejamento educacional apropriado às necessidades do superdotado, bem como na provisão de estratégias de apoio psicossocial a esse aluno.

O conhecimento acerca de suas potencialidades e limitações poderá contribuir para subsidiar a escola no que diz respeito à oferta de estratégias adequadas ao desenvolvimento de talentos e de uma aprendizagem significativa.

Este estudo foi realizado com o intuito de verificar as diferenças entre alunos superdotados acelerados (grupo I), não superdotados (grupo II) e superdotados não acelerados (grupo III) em relação à inteligência, personalidade e criatividade, assim como investigar a relação entre essas variáveis. Espera-se, com isso, gerar reflexões acerca dos mitos que permeiam a prática da aceleração escolar e, ainda, chamar atenção para as características particulares de indivíduos talentosos que, muitas vezes, sofrem com os estereótipos atribuídos aos indivíduos muito criativos e com inteligência acima da média, como o de desajustamento emocional.

\section{Método}

\section{Participantes}

Esta pesquisa foi realizada com 30 estudantes do ensino médio e superior - 15 do sexo feminino e 15 do sexo masculino -, com idades entre 15 e 20 anos $(M=17,3$ anos; $D P=1,57)$. Eles foram divididos em três grupos e selecionados de acordo com 
diferentes critérios que serão descritos a seguir. Destaca-se que para todos os grupos foi utilizada uma amostra de conveniência.

\section{Grupo I}

Os participantes deste grupo foram selecionados de acordo com os seguintes critérios: a) ter sido aprovado em uma instituição pública de ensino superior antes da conclusão formal do ensino médio (i. e., aceleração mediante entrada precoce na universidade) e b) estar cursando o primeiro, segundo ou terceiro semestre do curso. Participaram desse grupo dez estudantes (sendo cinco do sexo feminino e cinco do sexo masculino) de uma universidade federal da região centro-oeste, matriculados nos cursos de psicologia $(\mathrm{n}=3)$, economia $(n=1)$, direito $(n=1)$, farmácia $(n=1)$, estatística $(n=1)$, ciência da computação $(n=1)$, relações internacionais $(n=1)$ e engenharia mecatrônica $(n=1)$, com idades entre 16 e 20 anos $(M=17,7, D P=1,15)$.

Um estudante declarou ter sido acelerado durante sua trajetória escolar na educação básica e dois relataram ter frequentado atendimento educacional especializado - classe especial dentro da escola regular, indicada para os melhores alunos em determinadas disciplinas. Questionados se já haviam feito algum tipo de acompanhamento psicológico, metade afirmou que sim e a outra metade que não. Com relação à posição na família, seis participantes eram primogênitos ou filhos únicos e quatro se declararam como filhos do meio ou mais novos. Em consonância com a definição de superdotação acadêmica, mencionada anteriormente e ancorada no Modelo dos Três Anéis (Renzulli, 2016b), considerou-se que os integrantes desse grupo apresentavam características de superdotação em função de terem sido acelerados.

\section{Grupo II}

Este grupo foi utilizado como controle e, para a seleção dos participantes, foram estabelecidos três critérios: a) ter sido aprovado em uma instituição pública de ensino superior apenas após a conclusão formal do ensino médio; b) estar cursando o primeiro, segundo ou terceiro semestre do curso e c) ter no máximo 20 anos. $O$ critério de idade máxima foi acrescido para que a média de idade ficasse o mais próxima possível dos outros grupos.

Participaram desse grupo dez estudantes (quatro do sexo masculino e seis do sexo feminino) de uma universidade federal da região centro-oeste, matriculados nos cursos de psicologia $(n=7)$, história $(n=1)$, biotecnologia $(n=1)$ e estatística $(n=1)$, com idades entre 18 e 20 anos $(M=18,6$ anos; $D P=0,84)$. Questionados se já haviam feito algum tipo de acompanhamento psicológico, apenas três participantes afirmaram que sim. Com relação às trajetórias escolares, todos afirmaram que nunca foram acelerados durante o percurso escolar e que não participaram de nenhum atendimento educacional especializado. No tangente à posição na família, sete participantes afirmaram ser o filho mais novo ou do meio, e três, o primogênito.

\section{Grupo III}

Este grupo foi estabelecido como um segundo grupo controle, cuja participação incluiu os seguintes critérios: a) ser estudante do ensino médio e b) frequentar um Atendimento Educacional Especializado para Alunos com Altas Habilidades/Superdotação. Assim, o grupo contou com dez participantes (quatro do sexo feminino e seis do sexo masculino), com idades entre $15 \mathrm{e}$ 17 anos $(M=15,6$ anos; $D P=0,84)$, identificados como talentosos nas áreas de ciências exatas $(n=7)$ e ciências humanas $(n=3)$. O tempo médio de participação no programa foi de três anos, variando de 1 a 6 anos.

Questionados se já haviam feito algum tipo de acompanhamento psicológico para além da avaliação pela psicóloga do programa, metade afirmou que sim e a outra que não. Quatro desses alunos são da primeira série do ensino médio, quatro da segunda e dois da terceira série. Apenas uma pessoa afirmou ter sido acelerada durante sua trajetória escolar. No tangente às aspirações para o ensino superior, todos afirmaram ter interesse em ingressar em alguma instituição de nível superior, porém também declararam que nunca foram aprovados em nenhuma seleção de ingresso até o momento da coleta de dados.

Com relação à posição na família, a maioria declarou ser o primeiro filho $(n=6)$ ou filho único $(n=1)$, e três estudantes afirmaram ser filhos mais novos. Esse grupo foi escolhido como grupo de comparação, tendo em vista sua similaridade com o perfil de estudantes universitários ingressantes precocemente na universidade. Para além da faixa etária semelhante, infere-se que os estudantes de ambos os grupos apresentam características de superdotação, seja em função da participação em atendimento especializado ou pela aprovação em uma concorrida seleção de ingresso à universidade pública antes de 
concluir formalmente os estudos no ensino básico. No entanto, para além das semelhanças, esse grupo permitirá comparar as diferenças entre os grupos de alunos superdotados acelerados e não acelerados.

\section{Instrumentos}

Para além de um questionário sociodemográfico, foram utilizados quatro testes psicológicos, sendo um de inteligência, um de criatividade e dois de personalidade. Foram escolhidos dois testes de personalidade pelo fato de utilizarem metodologias distintas para mensurar o construto e focarem em diferentes dimensões do fenômeno.

\section{Matrizes Progressivas de Raven - Escala geral}

Trata-se de um teste de inteligência não verbal, que pode ser aplicado de forma individual ou grupal e não tem limitação de tempo. É composto pelas escalas infantil, geral e avançada. Neste estudo, optou-se por utilizar a escala geral, uma vez que abrange todas as idades e níveis de ensino e pelo fato de a pesquisa não estabelecer como critério de inclusão dos participantes a apresentação de inteligência superior ou média. O resultado oferece sete possibilidades de classificação da inteligência, que variam de inteligência superior a indício de deficiência mental. Conforme Campos, "as Matrizes são um instrumento válido para apurar a capacidade atual de uma pessoa para pensar claramente e realizar um trabalho intelectual preciso" (2002, p. 10). Raven (2012) enfatiza que esse é um teste de observação e clareza de pensamento, não sendo correto afirmá-lo como um teste de inteligência geral. O índice de fidedignidade varia de 0,83 a 0,93.

\section{Avaliação da criatividade por palavras - Teste de Torrance versão brasileira}

Esse instrumento é uma tradução e adaptação brasileira do Teste Torrance de Pensamento Criativo (TTPC) (Torrance, 1966), realizada por Wechsler (2004). OTTPC é um dos instrumentos mais utilizados no Brasil e no mundo para avaliação desse construto (Remoli \& Capellini, 2017), e "permite avaliar algumas das dimensões relacionadas ao processo criativo e à personalidade criativa por meio de indicadores expressos em forma de palavras" (Wechsler, 2004, p. iii). Apesar de o instrumento contar com as formas verbal e figurativa, neste estudo, optou-se pela utilização apenas da primeira, de forma a não cansar os participantes, tendo em vista a quantidade e a duração da aplicação dos instrumentos selecionados para a coleta de dados.

$\mathrm{O}$ teste pode ser aplicado de forma individual ou grupal e é composto por seis atividades com tempo limitado para a realização de cada uma, totalizando 40 minutos. Ele avalia os seguintes fatores: fluência, flexibilidade, originalidade, elaboração, fantasia, perspectiva incomum, expressão de emoção e uso da analogias/metáforas. Ao final, gera os Índices Criativos Verbais 1 - soma dos quatro primeiros fatores - e 2 soma dos oito fatores -, e se estabelece a classificação da criatividade entre superior, acima da média, média, abaixo da média e inferior. A autora reporta níveis satisfatórios de precisão do teste.

\section{Rorschach-Sistema compreensivo}

Trata-se de um teste projetivo de personalidade muito útil para avaliar aspectos relativos à organização e ao funcionamento da estrutura de personalidade (Exner, 1999a), mas também conta com bons indicadores do potencial intelectual e criativo (Sendín, 1999a). O teste deve ser aplicado individualmente e não tem limite de tempo.

Para a realização deste estudo, foram escolhidos três índices e quatro variáveis identificadas por outras pesquisas como bons indicadores de inteligência e criatividade (Ferracuti, Cannoni, Burla, \& Lazzari, 1999; Sakamoto, Lapastini, \& Silva, 2003; Schwartz \& Canetti, 2014), a saber:

a) DEPI: Índice de Depressão - se atingir um valor igual ou maior que cinco, a constelação é considerada positiva, o que indicaria fortemente a presença de comportamentos depressivos no sujeito (Sendín, 1999b);

b) CDI: Índice de Déficit Relacional - se atingir um valor igual a quatro ou cinco, a constelação é considerada como positiva, o que indicaria certa dificuldade em lidar com as demandas do meio social (Sendín, 1999c);

c) INTEL: apesar de ainda não ser considerada uma constelação formal no sistema compreensivo de Rorschach, Sendín (1999b) sugere que existe um conjunto de variáveis que, ao aparecerem juntas em um protocolo, representam boas capacidades intelectuais do sujeito. Segundo pesquisa de Sendín e García Alba (citado por Sendín, 1999c), essas variáveis estavam presentes em $75 \%$ dos sujeitos com QI superior e ausente em $77,7 \%$ dos que tinham QI médio ou abaixo da média. Res- 
salta-se que, por ainda não estar formalizada, a constelação não tem um nome, tendo sido assim nomeada pelas pesquisadoras;

d) FQu: Qualidade Formal (Form Quality) - indica respostas pouco frequentes, mas que não violam significativamente os contornos da forma;

e) S: É um código acrescido quando uma resposta é dada utilizando-se uma área de espaço em branco da mancha. Dependendo do grau dessas respostas, poderá indicar ou "uma busca de independência e auto-afirmação. . . ou um estilo de oposição e negativismo" (Sendín, 1999b, p. 132);

f) DQ: a Qualidade Evolutiva (Developmental Quality) é um código utilizado para informar sobre a qualidade de análise e síntese do sujeito (Sendín, 1999a). Quando é sintetizada (DQ+), significa que o sujeito entendeu dois objetos separados como relacionados, o que seria um indicativo de criatividade e inteligência (Exner, 1999b; Schwartz \& Canetti, 2014; Sendín, 1999a);

g) R: representa o número total de respostas dadas pelo sujeito em um protocolo. "Um número elevado de respostas significa capacidade para ser produtivo e, portanto, é um índice de bom potencial intelectual" (Sendín, 1999c, p. 43).

\section{Inventário Fatorial de Personalidade (IFP-II)}

O Inventário Fatorial de Personalidade (IFP-II) fundamenta-se na Teoria das Necessidades, de Henry Murray. O IFP-II avalia 13 fatores de personalidade (assistência, intracepção, afago, deferência, afiliação, dominância, desempenho, exibição, agressão, ordem, persistência, mudança, autonomia), totalizando 100 itens dispostos em escala do tipo likert, na qual 1 corresponde a "nada característico" e 7 a "totalmente característico". Três fatores de segunda ordem - a) Necessidades afetivas, b) necessidades de organização, e c) necessidades de controle e oposição - foram gerados a partir do agrupamento dos itens, conforme a análise de rotação varimax e normalização Kaiser (Leme, Rabelo, \& Alves, 2013). O teste pode ser aplicado de forma individual ou grupal e não tem limite de tempo (Ambiel, 2013). Os fatores medidos pelo instrumento obtiveram um alfa de Cronbach de pelo menos 0,69.

\section{Procedimentos}

O projeto de pesquisa foi submetido e aprovado pelo Comitê de Ética em Pesquisa do Instituto de Ciências Humanas de uma universidade da região centro-o- este, com o parecer no 2.712 .606 , e a coleta de dados do grupo III nas salas do Atendimento Educacional Especializado para Alunos com Altas Habilidades/Superdotação foi autorizada pela instituição responsável.

Foi feito contato com as professoras do atendimento para agendamento da aplicação dos instrumentos e para fornecer os Termos de Consentimento Livre e Esclarecido (TCLE) aos responsáveis e os Termos de Assentimento Livre e Esclarecido (TALE) aos alunos para serem assinados. O mesmo procedimento foi seguido com relação aos grupos I e II, com os quais a pesquisadora entrou em contato para convidá-los a participar da pesquisa e agendar, caso aceitassem, as datas para aplicação dos instrumentos conforme a disponibilidade do participante. Aos menores de idade, foi enviado previamente um TCLE para ser assinado por um responsável e levado no primeiro dia da coleta.

A aplicação dos instrumentos ocorreu em dois dias, com duração de aproximadamente 2 horas em cada um. Para a maioria dos integrantes, a coleta ocorreu de forma individual. Porém, quando foi possível agendar para o mesmo horário, a aplicação se deu coletivamente, dado que os manuais preveem essa possibilidade - com exceção do Rorschach, que foi aplicado individualmente em todos os participantes. Ao final do segundo dia de coleta, perguntou-se aos participantes se eles teriam indicações de colegas que se encaixassem nos critérios de cada grupo para participar da pesquisa.

\section{Análise de dados}

Os dados foram processados pelo programa SPSS (Statistical Package for Social Sciences) por meio de análises estatísticas descritivas e inferenciais. Ao efetuar o teste Kolmogorov-Smirnov de normalidade da amostra e o teste Levene de homogeneidade das variâncias, foi possível verificar que algumas das variáveis não estavam distribuídas de maneira normal e não eram homogêneas. Segundo Marôco (2018), no caso de não cumprimento de ao menos um dos pressupostos para o uso de testes paramétricos (normalidade, homogeneidade e linearidade da amostra), e ainda no caso de amostras pequenas, sugere-se que a análise não paramétrica dos dados seja mais adequada e eficaz. Por isso, com a intenção de comparar as variáveis independentes (inteligência, criatividade e personalidade) em relação às variáveis dependentes (grupos I, II e III), foram utilizados os testes de Kruskal-Wallis e Mann-Whitney - alternativas não paramétricas para a análise de vari- 
ância univariada (ANOVA) e o teste $t$, respectivamente (Marôco, 2018). Para investigar a relação entre as variáveis, foi utilizada a análise de correlação não paramétrica de Spearman que, segundo Barbetta (2008), é uma boa alternativa no caso de amostras pequenas e que não cumprem os requisitos de normalidade.

\section{Resultados}

A partir das análises descritivas, observou-se que grupo I obteve uma média no teste de inteligência de $57,2(D P=2,29)$, correspondente, segundo o manual do instrumento, à classificação de inteligência definidamente superior à média. Nos alunos do grupo II, a média do escore bruto de inteligência foi de 53,6 $(D P=3,40)$, correspondente à classificação de inteligência mediana, e os alunos do grupo III alcançaram média de $54,4(D P=3,71)$, o que se enquadra na classificação de inteligência superior à média. Para comparar se havia alguma diferença significativa do ponto de vista estatístico entres os três grupos, foi feita uma análise de Kruskal-Wallis, a qual apontou diferença moderadamente significativa entre os grupos $\left(X^{2}[2, \mathrm{~N}=30]=5,975 ; p=0,05\right)$. Para identificar entre quais grupos estava essa diferença, foi feita uma análise de Mann-Whitney com os grupos pareados, que demonstrou que a diferença significativa estava entre os grupos I e II ( $U=19,0, z=-2,359, p=0,019$ ). Os participantes do grupo I obtiveram média mais elevada em comparação à do grupo II. Entre os outros grupos, a análise não apontou resultados significativos.

Em relação à criatividade, as pontuações médias alcançadas pelos grupos I e III nos Índices Criativos Verbal 1 e 2 correspondem à criatividade acima da média, enquanto a do grupo II, para ambos os índices, é de criatividade média. Ao efetuar a análise de Kruskal-Wallis, apenas "fluência" $\left(X^{2}[2, \mathrm{~N}=30]=6,302\right.$; $p=0,043)$ e "flexibilidade" $\left(X^{2}[2, \mathrm{~N}=30]=6,415\right.$; $p=0,040)$ demonstraram resultado significativo, sugerindo que haveria diferença entre os grupos.

Para identificar entre quais grupos estaria situada a diferença, foi feita a análise de Mann-Whitney par a par entre eles. Em relação à fluência, houve diferença significativa entre os grupos I e II $(U=20,000$; $Z=-2,273 ; p=0,023$ ), com os participantes do grupo I apresentando média mais elevada, e entre os grupos II e III ( $U=23,000 ; Z=-2,043 ; p=0,041)$, revelando média superior do grupo III em relação ao II. Já em relação à flexibilidade, a diferença foi percebida apenas entre os grupos I e II ( $U=16,500 ; Z=-2,542 ; p=0,011)$, e o pri- meiro grupo obteve desempenho superior. A análise de Mann-Whitney também foi realizada nos casos em que o resultado de significância, no teste de Kruskal-Wallis, ficou próximo a $p=0,05$, pois uma vez que essa análise não permite verificar a diferença entre grupos dois a dois, hipotetizou-se que, por $p$ estar próximo a um resultado significativo, poderia haver alguma diferença entre os grupos, se analisados par a par. A partir dessas análises, foi possível perceber que houve diferença significativa entre os grupos I e II com relação aos Índices Criativos Verbais $1(U=18,500, Z=-2,382$, $p=0,017)$ e $2(U=18,000, Z=-2,420, p=0,016)$. Os participantes do grupo I apresentaram médias mais elevadas em comparação às do grupo II.

Com relação à personalidade, percebe-se, a partir da análise descritiva dos escores brutos das variáveis do Inventário Fatorial de Personalidade, que não houve diferenças significativas entre as médias dos grupos. O teste de Kruskal-Wallis confirma essa análise, pois demonstrou que tampouco houve diferenças significativas entre os grupos do ponto de vista estatístico. Porém, de acordo com a Tabela 1, destaca-se que em relação aos fatores de persistência, ordem e o fator de segunda ordem 2 , referente às necessidades de organização, os três grupos obtiveram pontuações referentes a um escore extremamente baixo, fraco ou médio fraco. Já em relação aos fatores de desempenho, dominância, autonomia, intracepção, exibição, agressão e ao fator de segunda ordem 3, correspondente às necessidades de controle e oposição, os três grupos obtiveram pontuações referentes a escores médio fortes, fortes ou extremamente altos. $\mathrm{O}$ fator de segunda ordem 1 , referente às necessidades afetivas, não obteve correspondência em nenhum dos grupos, recebendo classificação de escore médio no grupo I, forte no grupo II e fraco no grupo III.

Apesar de os dados obtidos pela análise de Kruskal-Wallis demonstrarem que, entre os grupos, não há nenhuma diferença significativa do ponto de vista estatístico com relação às variáveis medidas pelo teste de Rorschach, a análise descritiva das variáveis do teste evidenciou que, com relação à DEPI, seis alunos dos grupos I e III alcançaram um escore de cinco pontos ou mais, fazendo com que a constelação fosse positivada; do grupo II, foram sete alunos. Em relação ao $\mathrm{CDI}$, seis alunos do grupo I obtiveram o índice positivo, cinco do grupo II e quatro do grupo III. Por último, em relação à INTEL, obtiveram índices positivos dois alunos dos grupos I e III e nenhum aluno do grupo II. 
Tabela 1

Classificação dos fatores de personalidade para cada grupo.

\begin{tabular}{|c|c|c|}
\hline \multirow{3}{*}{ Grupo I } & Fracos & Fator de segunda ordem 2, persistência, deferência, afiliação, ordem. \\
\hline & Médios & Fator de segunda ordem 1, assistência, afago. \\
\hline & Fortes & $\begin{array}{l}\text { Fator de segunda ordem 3, desempenho, dominância, mudança, autonomia, intracepção, } \\
\text { exibição, agressão. }\end{array}$ \\
\hline \multirow{3}{*}{ Grupo II } & Fracos & Fator de segunda ordem 2, persistência, ordem. \\
\hline & Médios & Mudança, deferência, afiliação. \\
\hline & Fortes & $\begin{array}{l}\text { Fatores de segunda ordem } 1 \text { e 3, desempenho, dominância, autonomia, intracepção, } \\
\text { exibição, agressão, afago, assistência. }\end{array}$ \\
\hline \multirow{3}{*}{ Grupo III } & Fracos & Fatores de segunda ordem 1 e 2, persistência, deferência, ordem, deferência, afiliação. \\
\hline & Médios & Mudança, assistência, afago. \\
\hline & Fortes & Fator de segunda ordem 3, desempenho, dominância, autonomia, intracepção, exibição. \\
\hline
\end{tabular}

Fracos = escores extremamente baixos, fracos e médio fracos. Médios = escores médios. Fortes = escores extremamente altos, fortes e médio fortes.

Para investigar a relação entre as variáveis independentes, foi feita uma análise de correlação de Spearman. A partir disso, pôde-se observar que, com relação à variável de inteligência, houve uma correlação positiva significativa apenas com as variáveis "analogia" ( $\left.r_{s}=0,426 ; p=0,019\right)$, do teste de criatividade, e "agressão" ( $\left.r_{s}=0,367 ; p=0,046\right)$, do IFP-II. Com relação às variáveis de personalidade aferidas pelo IFP-II, apenas "agressão", "dominância", "desempenho" e "intracepção" obtiveram correlações significativas com pelo menos uma variável medida pelos outros instrumentos. "Agressão" demonstrou correlação positiva com "inteligência" ( $\left.r_{s}=0,367 ; p=0,046\right)$ e correlações negativas com "elaboração" $\left(r_{s}=-0,362\right.$; $p=0,049)$ e "fantasia" $\left(r_{s}=-0,372 ; p=0,043\right)$. "dominância" correlacionou-se, de forma positiva, apenas com "perspectiva incomum" $\left(r_{s}=0,375 ; p=0,041\right)$, ao passo que "desempenho" apresentou correlação negativa apenas com DEPI $\left(r_{s}=-0,417 ; p=0,022\right)$. Por último, "intracepção" apresentou correlações positivas significativas com $\mathrm{FQu}\left(r_{s}=0,458 ; p=0,011\right)$, $\mathrm{S}\left(r_{s}=0,381, p=0,038\right)$ e R $\left(r_{s}=0,429 ; p=0,018\right)$. Vale a pena destacar que, apesar de INTEL não ter se correlacionado significativamente com nenhuma variável dos outros testes, entre os índices do Rorschach, a constelação correlacionou-se de maneira significativa e negativa com CDI $\left(r_{s}=-0,470, p=0,009\right)$ e positiva com S $\left(r_{s}=0,399 ; p=0,029\right), \mathrm{DQ}+\left(r_{s}=0,660 ; p=0,000\right)$, $\mathrm{S}\left(r_{s}=0,645 ; p=0,000\right)$ e R $\left(r_{s}=0,370 ; p=0,044\right)$. Ressalta-se, ainda, as correlações positivas de DQ+ com as variáveis de criatividade "elaboração" $\left(r_{s}=0,412\right.$; $p=0,024)$, "originalidade" $\left(r_{s}=0,469 ; p=0,009\right)$ e os Índices Criativos Verbais $1\left(r_{s}=0,407 ; p=0,026\right)$ e 2 $\left(r_{s}=0,457 ; p=0,011\right)$.

\section{Discussão}

Considerando as controvérsias acerca dos temas inteligência, criatividade e personalidade de indivíduos com comportamentos de superdotação, este estudo teve como objetivo investigar a relação entre essas variáveis em alunos superdotados acelerados, não acelerados e não superdotados, bem como investigar a relação entre as variáveis em si. Ressalta-se que a avaliação se deu por meio de instrumentos psicométricos específicos, de modo que foi feito uso das mesmas referências teóricas utilizadas em seus respectivos construtos.

Para a realização desta pesquisa, utilizou-se como um dos critérios para inferência de comportamentos de superdotação a aprovação do aluno em uma concorrida seleção de ingresso à universidade pública antes da conclusão formal dos estudos no ensino básico (grupo I). Essa suposição pôde ser confirmada pelo fato de os alunos desse grupo, além de terem sido acelerados do ensino básico para o ensino superior, terem obtido as maiores médias, tanto no teste de inteligência quanto no de criatividade. Além disso, foram encontrados, nesse grupo, alunos que já haviam passado pela aceleração de estudos no ensino básico e/ou por algum programa de educação especializada para alunos mais avançados em uma ou mais áreas, não tendo sido encontradas pessoas com esse perfil no outro grupo de alunos universitários. 
Apesar de esses fatos não serem determinantes para a identificação de um aluno como AH/SD, a literatura da área aponta que a aceleração de estudos e a participação em salas de ensino especiais são muito comuns e indicadas para atender a alunos com esse perfil (Reis et al., 2016; Renzulli, 2016a).

Além disso, a literatura aponta que o superdotado é, geralmente, o filho mais velho ou o primogênito da família (Aspesi, 2007; Neves-Pereira, 2007). Isso está de acordo com o perfil de estudantes superdotados encontrado neste estudo, no qual $65 \%$ dos alunos dos grupos I e III declararam ser filhos únicos ou primogênitos, enquanto os do grupo II foram apenas $30 \%$.

Muitas controvérsias são tecidas em torno do tema da aceleração de estudos, principalmente no tangente às questões de ajustamento social e emocional. No entanto, como afirmam Brody et al. (2004), Steenbergen-Hu e Moon (2011) e Maia-Pinto e Fleith (2012), a maior parte das pesquisas sugere que os alunos acelerados não enfrentam mais problemas socioemocionais do que seus pares não superdotados. Os resultados desta investigação vão ao encontro de tais achados, tendo em vista que não foram identificadas diferenças significativas do ponto de vista estatístico nos testes de personalidade em relação aos grupos I e III, e tampouco foram encontradas grandes diferenças com relação à média dos escores brutos. Além disso, apesar de não terem sido identificadas diferenças significativas no tangente à inteligência entre os grupos I e III, destaca-se que o grupo I obteve maiores médias no escore bruto com relação a essa medida. Levanta-se a hipótese de que, com uma amostra maior, os dados iriam ao encontro do que afirmam Reis et al. (2016) acerca do melhor rendimento acadêmico encontrado nos alunos cujos currículos foram compactados.

Estudos teóricos e empíricos (Gagné, 2005; Gonçalves \& Fleith, 2011; Macedo et al., 2017; Renzulli, 1978, 2016a) revelam que os alunos superdotados têm maiores escores de inteligência e criatividade em relação a seus pares não superdotados. Os resultados da presente pesquisa corroboram esses achados, uma vez que os grupos de alunos superdotados obtiveram classificação de inteligência e criatividade superior à média, ao passo que as médias dos alunos não superdotados apontaram inteligência e criatividade medianas. Gonçalves e Fleith (2011), em contrapartida, não encontraram diferenças entre alunos superdotados e não superdotados no construto de inteligência, mensurado por meio do teste das Matrizes Progressivas de Raven.
No entanto, apesar de o escore bruto de inteligência dos grupos I e III ter sido superior ao do grupo II, apenas a média do grupo I demonstrou ser significativamente superior, do ponto de vista estatístico, em relação à do grupo II. Inicialmente, esperava-se que o grupo III, por ser formado por alunos superdotados, também obtivesse média significativamente superior em relação ao grupo II., mas é preciso lembrar que, conforme Campos (2002), o Teste das Matrizes Progressivas de Raven "pretende abranger toda a amplitude do desenvolvimento intelectual mais do que diferenciar claramente entre os indivíduos" (p. 7). Tendo em vista que o instrumento de inteligência utilizado nesta pesquisa mensura apenas o pensamento analítico e indutivo (Gonçalves \& Fleith, 2011; Muniz \& Lins, 2018), pode-se supor que alunos dos grupos II e III se diferenciariam mais em outros testes de inteligência que avaliem outras facetas do construto. Por outro lado, apesar de o índice de inteligência de Rorschach (INTEL), que "possui um elevado poder discriminador do nível de desenvolvimento cognitivo" (Sendín, 1999b, p. 44), não ter apresentado diferença estatisticamente significativa entre os grupos, ele só foi positivado por alunos dos grupos I e III. Isso indica a possibilidade de os alunos superdotados apresentarem maior capacidade de processamento de dados e adaptação com adequação razoalmente convencional, ou seja, maior desenvolvimento cognitivo em termos qualitativos (Sendín, 1999b).

Com relação à criatividade, Gonçalves e Fleith (2011) encontraram diferenças entre os grupos de superdotados e não superdotados apenas no tangente à originalidade. Esse resultado corrobora parcialmente os achados do presente estudo, no qual houve diferenças significativas com relação à criatividade, porém nos fatores de fluência, flexibilidade e no Índice Criativo Verbal 1, sendo que os grupos de alunos superdotados obtiverem maiores escores nos três fatores.

Esses resultados são convergentes com a literatura da área, que defende que os alunos superdotados têm, de modo geral, maior criatividade em relação a seus pares não superdotados (Renzulli, 2016b). Estudantes dos grupos I e III apresentam escores superiores aos do grupo II apenas no fator "fluência". Já nos fatores de flexibilidade e Índice Criativo Verbal 1, a diferença foi encontrada apenas entre os grupos I e II. Dado que flexibilidade envolve "a sensibilidade à mudança como a capacidade de se libertar de uma ideia inicial para explorar novas pistas" (Lubart, 2007, p. 21), esse resultado pode ser explicado pelo fato de 
o grupo I ter sido o único que apresentou um escore forte no fator "mudança" do IFP-II, e que escolheu a aceleração de estudos para o ensino superior, diante da opção de seguir com seus estudos no tempo convencional, tomando, assim, uma decisão disruptiva em relação aos participantes dos outros grupos.

Por fim, Gonçalves e Fleith (2011) não encontraram correlações significativas entre inteligência e criatividade mensuradas pelas Matrizes Progressivas de Raven e pelo Teste Torrance de Pensamento Criativo. Por outro lado, esta pesquisa, utilizando-se dos mesmos instrumentos psicométricos de inteligência e criatividade, demonstrou correlações significativas e positivas entre o escore bruto de inteligência e o fator de analogia de criatividade. Lubart (2007) afirma que analogia e metáfora são capacidades que compõem a inteligência sintética e analítica, além de capacidades intelectuais essenciais dentro do ato criativo. Dessa forma, a partir dos achados desta investigação, pode-se sugerir que a inteligência analítica seria um subconjunto da criatividade, corroborando os achados de Nakano (2012), que também encontrou relações significativas entre os dois construtos em seu estudo.

Inúmeras controvérsias existem acerca de se as AH/SD seriam um fator de risco para os indivíduos, sobretudo no sentido emocional e social. Porém, muitos estudos (Bergold, Wirthwein, Rost, \& Steinmayr, 2015; Francis, Hawes, \& Abbott, 2016) sugerem que os superdotados não apenas apresentam níveis de autoestima iguais aos de seus pares não superdotados, como, muitas vezes, apresentam melhor ajustamento social e emocional e menos problemas comportamentais. A correlação negativa encontrada entre INTEL e o Índice de Déficit Relacional (CDI) confirma essa hipótese, uma vez que sugere que quanto maior a inteligência, menores são as dificuldades de ajustamento social do indivíduo.

É importante destacar o fato de que, ainda que não tenham sido encontradas diferenças estatísticas significativas entre os grupos com relação aos fatores de personalidade do IFP-II, o único grupo que apresentou altos escores no fator de segunda ordem 1 - referente às necessidades afetivas - foi o grupo II, demonstrando que seriam pessoas com mais "necessidade de receber afeto, apoio e proteção de pessoas próximas, das quais espera ter seus desejos satisfeitos" (Ambiel, 2013, p. 50). Corrobora, portanto, Francis et al. (2016), que indicam que os superdotados têm certa vantagem no quesito emocional em relação a seus pares.
Além disso, um ajustamento emocional saudável foi percebido entre os alunos superdotados dos grupos I e III apontados pelos traços do IFP-II que indicam um sujeito imaginativo e fantasioso, aberto a novas experiências e mudanças, com grande senso de autoestima, autoconfiança e autonomia, ambicioso para realizar tarefas difíceis, que tende a quebrar padrões e desafiar as convenções e que gosta de ser valorizado por seu talento. Essas características identificadas no presente estudo estão em sintonia com resultados de estudos empíricos prévios (Wirthwein, Bergold, Preckel, \& Steinmayr, 2019; Zeidner \& Shani-Zinovich, 2011), os quais revelam que alunos superdotados têm maior abertura a novas experiências do que seus pares não superdotados.

Estão, também, de acordo com a literatura da área (Alencar, 2007; Brasil, 2006), segundo a qual esses traços são muito comuns nos indivíduos com comportamentos de AH/SD. A correlação positiva encontrada entre o fator intracepção (que diz respeito à tendência do sujeito de se levar pela fantasia, imaginação e pela procura à felicidade) e as variáveis FQu (maneira não convencional, porém aceitável, de ver a forma dos contornos da figura); $\mathrm{S}$ (que pode representar tanto "uma busca de independência e auto-afirmação ... [quanto] um estilo de oposição e negativismo" (Sendín, 1999b, p. 132)), e R (em que "um número elevado de respostas significa capacidade para ser produtivo e, portanto, é um índice de bom potencial intelectual" (Sendín, 1999b, p. 43)) também vai ao encontro desses resultados, sugerindo que um indivíduo fantasioso tende a apresentar, também, ideias originais, alta inteligência e um perfil mais independente e autônomo.

Uma das características descritas na literatura (Alencar, 2007; Ourofino \& Guimarães, 2007; Pfeiffer, 2013) presente em grande parte dos indivíduos com altas habilidades é a dificuldade de lidar com a frustração, o que se deve, muitas vezes, ao alto nível de perfeccionismo e autocobrança. Neste estudo, foi encontrada uma correlação negativa entre inteligência e o fator "agressão", do IFP-II, demonstrando que quanto mais altos forem os níveis de inteligência do indivíduo, maior será seu índice de agressão. Esse resultado vai ao encontro dos achados de Santos e Nascimento (2012), que identificaram correlações positivas entre os dois fatores. No entanto, como sugere o manual do IFP-II (Leme et al., 2013), altos escores no fator "agressão" podem estar relacionados à dificuldade em lidar com frustrações, 
como é exemplificado pelo item "às vezes, fico tão irritado(a) que sinto vontade de jogar e quebrar as coisas".

Por outro lado, "agressão" apresentou correlações negativas com os fatores de "elaboração" e "fantasia" do TTPC, sugerindo que uma vez que o indivíduo tem espaço para expressar sua criatividade, tende a demonstrar menos comportamentos agressivos. Sakamoto et al. (2003) afirmam que a utilização construtiva da agressividade e da assertividade é uma das características da pessoa criativa.

Ferracuti et al. (1999) realizaram uma pesquisa com o objetivo de observar se havia correlação entre as variáveis de criatividade mensuradas pelo TTPC e o Rorschach. Devido à significativa correlação encontrada entre criatividade e as variáveis S (resposta dada utilizando-se uma área de espaço em branco da mancha), FQu (Qualidade Formal) e DQ+ (Qualidade Evolutiva), concluíram que essas seriam as três variáveis do Rorschach mais associadas ao pensamento criativo. Esses resultados são compatíveis com os achados deste estudo, e chama-se atenção para o fato de que DQ+ correlacionou-se apenas com "elaboração" e "originalidade", indo de encontro à definição apresentada por Sendín (1999b) de que DQ+ está relacionado à capacidade de reorganizar o campo de estímulos a partir de um trabalho cognitivo elaborado.

A correlação negativa encontrada entre o Índice de Depressão e o fator Desempenho do IFP-II, que diz respeito à ambição de realizar uma tarefa difícil de maneira independente e com alto padrão de qualidade (Ambiel, 2013), destaca a importância ressaltada por muitos autores (Renzulli, 2016a; Sabatella \& Cupertino, 2007; Taslim \& Jabar, 2019) de um atendimento correto, estimulante e desafiador aos alunos. Sobretudo para os que apresentam comportamentos de AH/SD, pois sugere que quanto maior a chance de o indivíduo exercer atividades advindas da motivação intrínseca e ser livre para expressar e realizar suas ideias, menor o risco de ele desenvolver sintomas depressivos.

Na presente pesquisa, apesar de não terem sido encontradas diferenças significativas entre os grupos com relação aos índices de depressão e déficit relacional, houve mais alunos dos grupos de universitários com o Índice de Déficit Relacional positivado do que do grupo de alunos do ensino médio. Destaca-se, ainda, que $63 \%$ do número total de alunos obteve o índice de depressão positivado, indicando fortes indícios de estarem experienciando sintomas depressivos. Esse número é bastante expressivo, tendo em vista que, de acordo com a tabela normal para adultos não pacientes, a incidência de DEPI positiva é de apenas 3\% (Exner, 1999b).

Chama-se atenção para o fato de que apenas $43 \%$ do total de alunos afirmou estar fazendo ou já ter feito acompanhamento psicológico, sendo que o grupo II, que apresentou o maior número de alunos com DEPI positiva, foi também o com menos estudantes que fazem ou já fizeram acompanhamento psicológico. Destaca-se que a fase de transição do ensino médio para o ensino superior, assim como a adaptação à cultura universitária, tem sido descrita na literatura como um fator ansiogênico e estressor muito grande para os estudantes, inclusive para o superdotado, principalmente pelo afastamento dos familiares, maiores demandas e responsabilidades e contato social mais amplo e diversificado (Eisenberg, Gollust, Golberstein, \& Efner, 2007; Mortier et al., 2018; Pellá \& Magnabosco-Martins, 2018).

A correlação positiva encontrada entre o fator "perspectiva incomum" do TTPC e o fator "dominância" do IFP-II não estava sendo esperada a partir das hipóteses iniciais. Porém, tendo em vista que "perspectiva incomum" refere-se a "resistir às pressões da sociedade e a ter uma atitude inconformista perante fatos considerados, até então, como inquestionáveis" (Wechsler, 2004, p. 5), pode-se entender a dominância, que é definida como "desejo de controlar os outros, influenciar ou dirigir o comportamento deles através de sugestão, sedução, persuasão ou comando" (Ambiel, 2013, p. 84), como as diversas tentativas do indivíduo criativo de mostrar uma ideia controversa, ainda não aceita dentro dos padrões sociais vigentes.

\section{Conclusões}

Os resultados permitem concluir que existem diferenças entre alunos superdotados e não superdotados em relação à inteligência, tanto quantitativa quanto qualitativamente, sendo que os alunos com $\mathrm{AH} / \mathrm{SD}$ apresentaram maiores escores nessa medida. Da mesma forma, pode-se dizer que há diferenças entre alunos com e sem comportamentos de superdotação em relação à criatividade, sendo essa diferença expressa, principalmente, pelos fatores cognitivos desse construto. Além disso, pode-se concluir que, nessa pesquisa, inteligência e criatividade foram vistas como fatores diferentes, porém relacionados. Os resultados sugerem, ainda, a possibilidade de que inteligência analítica seja um subconjunto de criatividade.

Com relação à aceleração de estudos, os resultados apontaram que ingressar na universidade antes de 
concluir os estudos no ensino básico não foi maléfico para os estudantes, sugerindo que os alunos acelerados têm maiores índices de criatividade, inteligência e uma personalidade com maior abertura a novas experiências e mudanças. Por outro lado, apesar de não terem sido demonstradas fortes evidências nesse sentido, é possível que os alunos acelerados para o ensino superior estejam passando por maiores dificuldades de ajustamento social. Nesse sentido, ressalta-se a dificuldade de transição do ensino médio para o ensino superior - não apenas dos alunos acelerados e a necessidade de um acompanhamento mais próximo a esses estudantes.

Pode-se afirmar, ainda, que a depressão e os comportamento depressivos interferem no desempenho do aluno; mas, por outro lado, sugere-se que boas oportunidades de o aluno trabalhar com o que $o$ motiva e ter liberdade para criar e se desafiar também podem prevenir ou ajudar nos sintomas depressivos.

Este estudo permite pensar em novas possibilidades de avaliação e identificação de alunos superdotados. Segundo Brown et al. (2016), o processo de identificação é feito, principalmente, por meio de testes psicométricos de inteligência, criatividade e motivação, avaliação de portfólio e avaliação dos pais e professores. No entanto, como afirma Sakamoto et al. (2003), "o Rorschach com seu rico material interpretativo, pode contribuir muito na compreensão das dificuldades psicológicas apresentadas pelo indivíduo, assim como no discernimento de seu potencial de recursos afetivos, cognitivos e integradores-criativos" (p. 23). Ressalta-se ainda a contribuição trazida às pesquisas com a constelação de inteligência sugerida por Sendín (1999a), que ainda não foi oficialmente validada no Rorschach, mas demonstrou evidências de ser um bom indicador do potencial intelectual, sobretudo em termos qualitativos.

Como limitações deste estudo, ressaltam-se: a) o reduzido tamanho da amostra; b) amostra selecionada por conveniência, o que não permitiu grande variação de alunos por área de interesse; c) entrada precoce na universidade como um dos critérios utilizados para indicação de comportamentos de superdotação. Como sugestões para estudos futuros, pode-se destacar a possibilidade de: a) replicar esta pesquisa com uma amostra maior e mais diversificada de estudantes, em termos de áreas de interesses; b) realizar um estudo longitudinal com alunos que ingressaram precocemente na universidade, a fim de avaliar seu desempenho e desenvolvimento, e confirmar, com dados empíricos de rendimento acadêmico, a hipótese de que esses alunos possuem características de superdotação; c) realizar mais pesquisas no cenário brasileiro comparando alunos superdotados que passaram pela aceleração de estudos e alunos superdotados que não passaram.

\section{Referências}

Alencar, E. M. L. S. (2007). Características sócio-emocionais do superdotado: Questões atuais. Psicologia em Estudo, 12(2), 371-378. https://doi.org/10.1590/S1413-73722007000200018

Ambiel, R. A. M. (2013). Atualização dos estudos psicométricos em uma amostra brasileira. In I. F. A. S. Leme, I. S. Rabelo, \& G. A. S. Alves (Eds.), Inventário Fatorial de Personalidade (pp. 3579). Casa do Psicólogo.

Aspesi, C. C. (2007). A família do aluno com altas habilidades/superdotação. In D. S. Fleith (Ed.), A construção de práticas educacionais para alunos com altas habilidades/superdotação (vol. 2, pp. 2948). Ministério da Educação, Secretaria de Educação Especial.

Azevedo, S. M. L., \& Mettrau, M. B. (2010). Altas habilidades/superdotação: Mitos e dilemas docentes na indicação para o atendimento. Psicologia: Ciência e Profissão, 30(1), 3245. https://doi.org/10.1590/S141498932010000100004

Barbetta, P. A. (2008). Estatística aplicada às ciências sociais (7a ed.). Editora da UFSC.

Bergold, S., Wirthwein, L., Rost, D. H., \& Steinmayr, R. (2015). Are gifted adolescents more satisfied with their lives than their non-gifted peers? Frontiers in Psychology, 6, 110. https://doi.org/10.3389/fpsyg.2015.01623

Brasil, Ministério da Educação. (2006). Saberes e práticas da inclusão: Desenvolvendo competências para o atendimento às necessidades educacionais especiais de alunos com altas habilidades/superdotação. Secretaria de Educação Especial.

Brody, L. D., Assouline, S., \& Stanley, J. (1990). Five years of early entrants: Predicting successful achievement in college. Gifted Child Quartely, 34(4), 138142. https:// doi.org/10.1177/001698629003400402 
Brody, L. E., Muratori, M. C., \& Stanley, J. C. (2004). Early entrance to college: Academic, social, and emotional considerations. In N. Colangelo, S. G. Assouline, \& M. U. M. Gross (Eds.), A nation deceived: How schools hold back America's brightest students (vol. 2, pp. 97108). GERRIC.

Brown, S. W., Renzulli, J. S., Gubbins, E. J., Siegle, D., Zhang, W., \& Chen, C. H. (2016). Assumptions underlying the identification of the gifted and talented students. In S. M. Reis (Ed.), Reflections on gifted education (pp. 151-172). Prufrock Press.

Campos, F. (2002). Matrizes progressivas de Raven: Escala geral (5a ed.). CEPA.

Cross, J. R., \& Cross, T. L. (2015). Clinical and mental health issues in counseling the gifted individual. Journal of Counseling \& Development, 93(2), 163172. https://doi.org/10.1002/j.1556-6676.2015.00192.x

Cross, T. L., \& Andersen, L. (2016). Depression and suicide among gifted children and adolescents. In M. Neihart, S. I. Pfeiffer, \& T. L. Cross (Eds.), The social and emotional development of gifted children. What do we know? (pp. 79-90). Prufrock Press.

Eisenberg, D., Gollust, S. E., Golberstein, E., \& Efner, J. L. (2007). Prevalence and correlates of depression, anxiety, and suicidality among university students. American Journal of Orthopsychiatry, 77(4), 534542. https://doi. org/10.1037/0002-9432.77.4.534

Exner, J. E. (1999a). Validade e objetivos do Rorschach. In J. E. Exner, \& C. Sendín (Eds.), Manual de interpretação do Rorschach para o sistema compreensivo (pp. 1132). Casa do Psicólogo.

Exner, J. E. (1999b). Manual de classificação do Rorschach para o sistema compreensivo. Casa do Psicólogo.

Ferracuti, S., Cannoni, E., Burla, F., \& Lazzari, R. (1999). Correlations for the Rorschach with the Torrance testes of creative thinking. Perceptual Motor Skills, 89(3), 863870. https://doi.org/10.2466/pms.1999.89.3.863

Francis, R., Hawes, D. J., \&Abbott, M. (2016). Intellectual giftedness and psychopathology in children and adolescents: A systematic literature review. Exceptional Children, 82(3), 279302. https://doi.org/10.1177/0014402915598779

Gagné, F. (2005). From gifts to talents: The DMGT as a developmental model. In J. R. Sternberg, \& J. E. Davidson (Eds.), Conceptions of giftedness (2a ed., pp. 98119). Cambridge University Press.

Gardner, H. (1983). Frames of mind: The theory of multiple intelligences. Basics Books.

Gonçalves, F. C., \& Fleith, D. S. (2011). Estudo comparativo entre alunos superdotados e não-superdotados em relação à inteligência e criatividade. Psico, 42(2), 263268.

Guignard, J. H., Kermarrec, S., \& Tordjman, S. (2016). Relationships between intelligence and creativity in gifted and non-gifted children. Learning and Individual Differences, 52(1), 209215. https://doi.org/10.1016/j.lindif.2015.07.006

Ismail, M. J., Anuar, A. F., \&Yusuf, R. (2020). Exploring giftedness: Traits of cognitive and practical skills of a gifted child. International Journal of Education, Psychology and Counseling, 5(34), 189196. https:// doi.org/10.35631/ijepc.5340015

Leme, I. F. A. S., Rabelo, I. S., \& Alves, G. A. S. (2013). Aplicação, pontuação e interpretação. In I. F. A. S. Leme, I. S. Rabelo, \& G. A. S. Alves (Eds.), Inventário Fatorial de Personalidade (pp. 8186). Casa do Psicólogo.

Lubart, T. (2007). Psicologia da criatividade. Artmed.

Macedo, M. M. F., Mota, M. E., \& Mettrau, M. B. (2017). WISC-IV: Evidências de validade para grupos especiais de superdotados, 11(1), 6573.

Maia-Pinto, R. R., \& Fleith, D. S. (2012). Aceleración de la enseñanza para alumnos superdotados: Argumentos favorables y contrarios. Revista de Psicología, 30(1), 189214.

Marôco, J. (2018). Análise estatística com o SPSS statistics (7a ed.). ReportNumber.

Mortier, P., Cuijpers, P., Kiekens, G., Auerbach, R. P., Demyttenaere, K., \& Green, J. G. (2018). The prevalence of suicidal thoughts and behaviours among college students: A meta-analysis. Psychological Medicine, 48(4), 554565. https://doi.org/10.1017/S0033291717002215

Muniz, M., \& Lins, M. R. C. (2018). Avaliação da inteligência: Aspectos não verbais. In C. S. Hutz, D. R. Bandeira, \& C. M. Trentini (Eds.), Avaliação psicológica da inteligência e da personalidade (pp. 7388). Artmed.

Nakano, T. C. (2012). Criatividade e inteligência em crianças: Habilidades relacionadas? Psicologia: Teoria e Pesquisa, 28(2), 149159. https://doi.org/10.1590/S0102-37722012000200003

Neves-Pereira, M. S. (2007). Estratégias de promoção da criatividade. In D. S. Fleith (Ed.), A construção de práticas educacionais para alunos com altas habilidades/superdotação (vol. 2, pp. 1334). Ministério da Educação, Secretaria de Educação Especial. 
Nogueira, M., \& Fleith, D. S. (2019). Qual é a relação entre criatividade e transtorno mental? Revista Brasileira de Terapia Comportamental e Cognitiva, 21(3), 317331.

Ourofino, V. T. A. T., \& Guimarães, T. (2007). Características intelectuais, emocionais e sociais do aluno com altas habilidades/superdotação. In D. S. Fleith (Ed.), A construção de práticas educacionais para alunos com altas habilidades/superdotação (vol. 1, pp. 4152). Ministério da Educação, Secretaria de Educação Especial.

Pellá, L. M., \& Magnabosco-Martins, C. R. (2018).Vidas em formação: Narrativas de acadêmicos sobre a experiência na graduação em Psicologia. Psicologia, Diversidade e Saúde, 7(3), 385399. https://doi.org/10.17267/23173394rpds.v7i3.1936

Pfeiffer, S. I. (2013). Serving the gifted. Evidence-based clinical and psychoeducational practice. Routledge.

Raven, J. C. (2012). Matrizes Progressivas Escala Geral: Manual (5a ed.). Centro Editor de Psicologia Aplicada.

Reis, S. M., Westberg, K. L., Kulikowich, J. M., \& Purcell, J. H. (2016). Curriculum compacting and achievement test scores: What does the research say? In S. M. Reis (Ed.), Refletions on gifted education (pp. 271285). Prufrock Academic Press.

Remoli, T. C., \& Capellini, V. M. F. (2017). Relação entre criatividade e altas habilidades/superdotação: Uma análise crítica das produções de 2005 a 2015. Revista Brasileira de Educação Especial, 23(3), 455470. https://doi. org/10.1590/S1413-65382317000300010

Renzulli, J. S. (1978). What makes giftedness? Re-examining a definition. Phi Delta Kappan, 60(3), 180184. https:// doi.org/10.1177/003172171109200821

Renzulli, J. S. (2002). Emerging conceptions of giftedness: building a bridge to the new century. Exceptionality: A Special Education Journal, 10(2), 6775. https://doi.org/10.1207/S15327035EX1002_2

Renzulli, J. S. (2005). The three-ring conception of giftedness: A developmental model for promoting creative productivity. In R. J. Sternberg, \& J. Davidson (Eds.), Conceptions of giftedness (2a ed., pp. 246279). https://doi. org/10.1017/CBO9780511610455.015

Renzulli, J. S. (2016a). The enrichment triad model: A guide for developing defensible programs for the gifted and talented. In S. M. Reis (Ed.), Reflections on gifted education (pp. 193-210). Prufrock Academic Press.

Renzulli, J. S. (2016b). The three-ring conception of giftedness: A developmental model for promoting creative productivity. In S. M. Reis (Ed.), Refletions on gifted education (pp. 5590). Prufrock Academic Press.

Sabatella, M. L., \& Cupertino, C. M. B. (2007). Práticas educacionais de atendimento ao aluno com altas habilidades/ superdotação. In D. S. Fleith (Ed.), A construção de práticas educacionais para alunos com altas habilidades/ superdotação (vol. 1, pp. 6780). Ministério da Educação, Secretaria de Educação Especial.

Sakamoto, C. K., Lapastini, M. A. B., \& Silva, S. M. (2003). A criatividade no psicodiagnóstico de Rorschach: Uma possibilidade de enriquecimento à interpretação dos resultados. Psicologia: Teoria e Prática, 5(1), 1325.

Santos, M. T., \& Nascimento, E. (2012). Inteligência e personalidade: Um estudo correlacional em uma amostra de universitários. Estudos de Psicologia, 29(2), 163171. https://doi.org/10.1590/S0103-166X2012000200002

Schwartz, H., \& Canetti, L. (2014). Creativity and emotional distress on the Rorschach test. Rorschachiana, 35(1), 2341. https://doi.org/10.1027/1192-5604/a000043

Sendín, C. (1999a). Questões preliminares. In J. E. Exner, \& C. Sendín (Eds.), Manual de interpretação do Rorschach para o sistema compreensivo (pp. 3952). Casa do Psicólogo.

Sendín, C. (1999b). Traços afetivos. In J. E. Exner, \& C. Sendín (Eds.), Manual de interpretação do Rorschach para o sistema compreensivo (pp. 119162). Casa do Psicólogo.

Sendín, C. (1999c). Relações interpessoais. In J. E. Exner, \& C. Sendín (Eds.), Manual de interpretação do Rorschach para o sistema compreensivo (163171). Casa do Psicólogo.

Steenbergen-Hu, S., \& Moon, S. M. (2011). The effects of acceleration on high-ability learners: A meta-analysis. Gifted Child Quarterly, 55(1), 3953. https://doi.org/10.1177/0016986210383155

Sternberg, R. J. (1999). The theory of successful intelligence. Review of General Psychology, 3(4), 292316. https:// doi.org/10.1037/1089-2680.3.4.292

Sternberg, R. J. (2005). The WICS model of giftedness. In R. J. Sternberg, \& J. E. Davidson (Eds.), Conceptions of giftedness (2a ed., pp. 327342). Cambridge University Press.

Taslim, P. L., \& Jabar, C. (2019). Evaluation of acceleration program termination for gifted children learning needs. Advances in Social Science, Education and Humanities Research, 296, 114119. https://doi.org/10.2991/icsie-18.2019.22 
Torrance, E. P. (1966). Torrance Tests of Creative Thinking. Personnel Press.

Wechsler, S. M. (2004). Avaliação da criatividade por palavras: Teste de Torrance versão brasileira (2a ed.). Duo Paper.

Wirthwein, L., Bergold, S., Preckel, F., \& Steinmayr, R. (2019). Personality and school functioning of intellectually gifted and nongifted adolescents: Self-perceptions and parents' assessments. Learning and Individual Differences, 73(1), 1629. https://doi.org/10.1016/j.lindif.2019.04.003

Zeidner, M., \& Shani-Zinovich, I. (2011). Do academically gifted and nongifted students differ on the Big-Five and adaptive status? Some recent data and conclusions. Personality and Individual Differences, 51(5), 566570. https:// doi.org/10.1016/j.paid.2011.05.007

\section{Marina Nogueira}

Psicóloga clínica. Mestre em Processos de Desenvolvimento Humano e Saúde pela Universidade de Brasília. Brasília - DF. Brasil.

E-mail: marinanogueira@outlook.com

(1) https://orcid.org/0000-0003-3154-2358

Denise de Souza Fleith

Psicóloga. Pesquisadora do Conselho Nacional de Desenvolvimento Científico e Tecnológico (CNPq) e da Universidade de Brasília. Brasília - DF. Brasil.

E-mail: fleith@unb.br

(1) https://orcid.org/0000-0001-7512-8023

Este estudo foi realizado com apoio do Conselho Nacional de Desenvolvimento Científico e Tecnológico (CNPq), na modalidade de bolsa de mestrado.

Endereço para envio de correspondência:

Programa de Pós-Graduação em Psicologia do Desenvolvimento e Escolar. Instituto de Psicologia. Universidade de Brasília, Campus Darcy Ribeiro. CEP: 70910-900. Brasília - DF.

Recebido 27/01/2019

Aceito 23/06/2020

Received $01 / 27 / 2019$

Approved 06/23/2020

Recibido 27/01/2019

Aceptado 23/06/2020

Como citar: Nogueira, M., Fleith, D. S. (2021). Relação entre inteligência, criatividade, personalidade e superdotação no contexto escolar. Psicologia: Ciência e Profissão, 41 (n.spe 3), 1-16. https://doi.org/10.1590/1982-3703003219130

How to cite: Nogueira, M., Fleith, D. S. (2021). Association of intelligence, creativity, personality, and giftedness in the school context. Psicologia: Ciência e Profissão, 41 (n.spe 3), 1-16. https://doi.org/10.1590/1982-3703003219130

Cómo citar: Nogueira, M., Fleith, D. S. (2021). Relación entre inteligencia, creatividad, personalidad y superdotación en el contexto escolar. Psicologia: Ciência e Profissão, 41 (n.spe 3), 1-16. https://doi.org/10.1590/1982-3703003219130 